\title{
Schreiben, Diskursfähigkeit und Internet im fremdsprachlichen und muttersprachlichen Deutschunterricht
}

\section{Writing, discourse ability and internet in German as foreign und native language lessons}

\begin{abstract}
To teach effective writing skills is one of the tasks of German-as-native-language- and German-as-foreign-language lessons. Taking these as a basis, this paper aims at explaining different approaches, their advantages and disadvantages and at developing an new concept - writing as discourse ability. The paper emphasizes the necessity of this new concept, which enable the pupils to write "real" texts and take part In discourse. Furthermore it focuses on training program with internet.
\end{abstract}

KEYWORDS: Writing, German as native language, German as foreign language, discourse, essay writing, internet.

\section{EINLEITUNG}

Schreibenkönnen ist eine unabdingbare Voraussetzung der Sprachbeherrschung. Die Schreibfertigkeiten auszubilden, ist eine Aufgabe des Sprachunterrichts sowohl in der Muttersprache als auch in der Fremdsprache. Die muttersprachliche und die fremdsprachliche Didaktik gehen zwar von unterschiedlichen Voraussetzungen der Lernenden aus, anvisieren dennoch die Entwicklung gleicher Kompetenzen. Im Fall der Schreibfertigkeit handelt es sich um die Fähigkeit zum schriftlichen Gebrauch der Zielsprache in Wirklichkeitszusammenhängen und ihrer Bewältigung sowie um die Diskursfähigkeit, d.h. um die Fähigkeit solche Texte zu verfassen, mit denen 
sich ihre Autoren an den Diskursen der Öffentlichkeit beteiligen, sich in diese einschreiben und gegebenenfalls fortsetzen können. Dem Schreiben in der Fremdsprache kommen allerdings noch weitere zusätzliche Funktionen $\mathrm{zu}$ - Schreiben als Lernmedium - beispielsweise in (präkommunikativen) Aufgaben mit lernfördernder Wirkung oder bei der Leistungsüberprüfung. Zwischen beiden Didaktiken ergeben sich jedoch bei der Vermittlung dieser traditionell konzipierten Basisfertigkeit Wechselwirkungen. Ziel dieses Beitrags ist es, vor diesem Hintergrund ein Modellentwurf sowohl für den muttersprachlichen als auch für den fremdsprachlichen Schreibunterricht mit Einbeziehung der schriftlichen Wirklichkeit anvisierter Zielkultur und des Internets vorzustellen. Es soll jedoch keine konkrete Vorgehensweise aufgelegt werden, da thematische Aktualität und Spontaneität zu erhalten sind. Der Beitrag gliedert sich wie folgt. Zunächst wird auf die historische Entwicklung und gegenwärtige Wirklichkeit des Schreibunterrichts in der Muttersprache und in der Fremdsprache eingegangen. Daraufhin folgen die Begriffserklärungen von Text und Diskurs und in Anschluss ein Modell des Schreibunterrichts. Abgeschlossen wird der Beitrag mit einem praktischen Beispiel.

\section{SCHREIBEN IM MUTTERSPRACHLICHEN UND FREMDSPRACHLICHEN DEUTSCHUNTERRICHT}

Im Laufe der Geschichte erfuhr Schreiben im muttersprachlichen Deutschunterricht im schulischen Rahmen unterschiedliche Akzentuierungen. Das Augenmerk wurde auf Textformen und Schreibprodukte, auf Funktionen der Schriftstücke, auf Prozesse und Schreibstrategien und auf die Entfaltung der Persönlichkeit des Schreibenden gerichtet. In all diesen Konzepten klafften und tun dies immer noch die Schule und Wirklichkeit auseinander. An dieser Stelle sei in aller Kürze das Problem veranschaulicht. Der Aufsatzunterricht stellte die Textformen und die Schriftstücke in den Vordergrund. Er erschöpfte sich in der Vermittlung von Normen, Formen und Konventionen schriftlicher Kommunikation (Marthaler 1962; Ludwig 1988; 2003). Der freie Aufsatz kritisierte eine strenge Instruktion, aber auch in diesem Ansatz wurden nach einer anfänglichen Euphorie und Darstellung der Schüler als kleine Künstler Schriftstücke verfasst, die sich nach Idealen der schulischen Realität richteten (Karg 2007). Ein neues Konzept vom Schreibunterricht wurde in Theorien des kommunikativen Schreibens geschaffen (Fritzsche 1980). Dieser Ansatz kam dem Ziel der schriftlichen Bewältigung der Situationen der Wirklichkeit am nächsten. Das Üben von Aufsatzformen trat in den Hintergrund, als bedeutend erschienen: die Situation, in der ein Text geschrieben wurde, die Intention des Schreibers hinsichtlich der Funktion bzw. Wirkung, die der zu schrei- 
bende Text haben sollte, und der Adressat, an den sich der Verfasser mit dem Text wendete. Das Ziel der Textproduktion wurde im Verfassen von solchen Texten gesehen, die von einem mit der Lehrperson nicht identischen Leser gelesen und verstanden werden können, und mit denen eine bestimmte $\mathrm{Ab}$ sicht verwirklicht wird. Das Konzept in seiner Gänze etablierte sich nicht, aber einzelne Aspekte, nämlich das Verfassen von pragmatisch orientierten Schriftstücken findet auch im gegenwärtigen Unterricht, besonders im fremdsprachlichen Deutschunterricht, seinen Platz. In den Ansätzen zum kreativen Schreiben beabsichtigten die Schreibdidaktiker den traditionellen schulischen Texten eine Absage zu erteilen (Spinner 1993; Brenner 1998; Fröchling 1990; Scheidt 1993). Die Reformer zielten auf die Identitätsfindung und innere Welt der Schreibenden ab. Die kreativen Verfahren wurden in den Rahmen des traditionellen Aufsatzes erfolgreich integriert. Exemplarisch sind hier einige Beispiele zu nennen. So wird das Sammeln von Ideen bei dem Verfassen einer Erzählung mittels Brainstorming unterstützt (Müller et al. 2005: 26, Kombi-Buch Deutsch 6. Schulbuch für den Unterricht. Bamberg: Buchner) oder zum Anlegen der Stoffsammlung beim Schreiben einer Inhaltsangabe sollen Cluster verwendet werden (Müller et al. 2006: 15, Kombi-Buch Deutsch 8. Schulbuch für den Unterricht. Bamberg: Buchner). Ein weiteres Beispiel ist das Sammeln von Argumenten mit Hilfe von Mind Map bei der Ausbildung der Fähigkeit, Sachverhalte zu erörtern (Biermann et al. 2000: 17, Deutschbuch 10, Arbeitsheft. Berlin: Cornelsen). Des Weiteren ist die Ideensammlung für das Verfassen von Erörterungen mit den Techniken des kreativen Schreibens Écriture automatique - zu nennen (Biermann et al. 1998: 49, Deutschbuch 10. Berlin: Cornelsen).

Das nachfolgende Konzept - die Prozessorientierung - fokussierte den Vorgang der Textproduktion und seine Phasen (Hayes, Flower 1980; Portmann 1991; Kruse, Perrin 2003).

Die Vorschläge von Schreibdidaktikern zur Optimierung des Schreibunterrichts, nämlich das Verlagern der Schreiborte aus dem Klassenzimmer, die Orientierung an der inneren Welt der Schreibenden, die Förderung ihrer Kreativität und Originalität, eine bewusste Gestaltung von Schreibsituationen, eine kontinuierliche Demonstration von Textmustern, Operatoren, und Funktionen, die ein Text erfüllt, die Anknüpfung an die unmittelbare Lebenswelt der Schüler, „Learning by doing“, Vermittlung von metakognitiven Kompetenzen, sind richtig. Sie haben bereits in den Rahmenrichtlinien ihre Verankerung gefunden, aber auch dadurch werden die Lernenden nicht ausreichend zu einem kompetenten schriftlichen Umgang mit den Situationen der Wirklichkeit befähigt. Das zu Schreibende bleibt abstrakt. Die Vorstellungen von den schulischen Texten werden auf abstrakte Schemen zurückgeführt, die wenig Ähnlichkeiten mit den Texten der Realität haben. Somit befinden sich die Schreibenden in einem kontinuierlichen Konflikt 
zwischen dem, was ihre eigenen Erfahrungen und Vorstellungen und die Wirklichkeit der schriftlichen Texte sind, und dem, was ihnen im schulischen Schreibunterricht abverlangt wird. Die gegenwärtige Situation im fremdsprachlichen Unterricht ist ähnlich.

Im Unterschied zum muttersprachlichen sah der fremdsprachliche Deutschunterricht weder bei der Grammatik-Übersetzungs- noch bei der direkten, der audiolingualen und der audiovisuellen Methode das Schreiben und die Ausbildung der Fertigkeit zum schriftlichen Ausdruck als eine seiner Aufgaben (vgl. Neuner, Hunfeld 2004; Huneke, Steinig 2002). Die Betrachtung des Schreibens als eine Hilfsfertigkeit beim Spracherwerb konnte nicht zur Fähigkeit der Lernenden, sich schriftlich auszudrücken, und mit den Wirklichkeitskontexten schriftlich kompetent umzugehen, führen. Erst im interkulturellen Ansatz begleitet mit der Übernahme der Vorstellungen aus der muttersprachlichen Didaktik vom Schreiben als einem Prozess wurde Schreiben als ein Bestandteil des Spracherwerbs eingeschätzt (Krumm 1994, Roche 2001). Allerdings wurde auch die Tradition des Verfassens von Texten nach abstrakten schematischen Verfahren, beispielsweise das Verfassen einer steigernden und einer dialektischen Erörterung transferiert. (Braun et al. 2000: 23, Unterwegs zur Vorbereitung auf die Zentrale Mittelstufenprüfung. Trainingsbuch. Berlin: Langenscheidt, Rohrmann et al. 2005: 11, Berliner Platz 1. Berlin: Langenscheidt, Rohrmann et al. 2004: 153, Berliner Platz 3. Berlin: Langenscheidt, Dallapiazza et al. 2003: 4, Tangram aktuell. Zertifikatsband. Ismaning: Hueber, Niebisch et al. 2009: 157, Schritte plus 1. Kursbuch und Arbeitsbuch mit Audio-CD zum Arbeitsbuch. Ismaning: Hueber, Köhl-Kuhn et al. 2008: 34, Mittelpunkt C1. Lehrbuch Deutsch als Fremdsprache für Fortgeschrittene. Stuttgart: Klett, Köhl-Kuhn et al. 2007: 117, Mittelpunkt B2. Lehrbuch Deutsch als Fremdsprache für Fortgeschrittene. Stuttgart: Klett, Dallapiazza et al. 2008: 85, Ziel B2, Bd. 1. Paket Kursbuch und Arbeitsbuch mit Lerner-Audio-CD/CD-ROM. Ismaning: Hueber.). Eine solche Unterrichtskonzeption befähigt jedoch nicht ausreichend zum schriftlichen Sprachgebrauch in außerunterrichtlichen Wirklichkeitssituationen und zu ihrer Bewältigung. Dieser verfahrenen Situation des muttersprachlichen und fremdsprachlichen Unterrichts könnte mit einer veränderten Vorgehensweise begegnet werden - nämlich der Einbeziehung des Diskurses in den Unterricht.

\section{BEGRIFFSERKLÄRUNGEN: DER TEXT UND DER DISKURS}

Einer der zentralen Begriffe in der Schreibdidaktik ist der Text. Was darunter zu verstehen ist, definiert Sprachwissenschaft. Allerdings wird diesem Terminus keine eindeutige Bedeutung zugewiesen. Die Schwierigkeiten 
bei der Textdefinition sind, wie Heinz Vater feststellt, teilweise objektiver, teilweise subjektiver Natur: „Sie liegen sowohl im Phänomen „Text" selbst begründet als auch in der Verschiedenheit der verwendeten Ansätze“ Vater (2002: 25). Die Definitionsschwierigkeiten resultieren darüber hinaus aus dem Wandel der Vorstellungen vom Text. Gegenwärtig verbreitet sich eine Vorstellung vom Text als einer vom Produzenten abgeschlossenen Ganzheit, die jedoch von anderen Texten nicht völlig unabhängig ist, sondern einen Bestandteil von übergreifenden Diskursen, in die er eingebettet ist, darstellt. Der Text ist danach einerseits eine zusammenhängende, in sich abgeschlossene und als solche identifizierbare Einheit des Sprachgebrauchs, ein "Ausschnitt aus diesem Universum" Adamzik (2004a: 255). Andererseits steht ein Text immer in einer Beziehung zu anderen, bereits vorhandenen Texten im Diskurszusammenhang.

Auch der Diskursbegriff kennt unterschiedliche Verwendungsweisen (vgl. dazu Stenschke 2005; Adamzik 2004a; 2004b; Bluhm et al. 2000). Busse und Teubert (1994: 14) definieren ihn aus linguistischer Perspektive

als eine Gesamtheit von mündlichen und schriftlichen Äußerungen zu einem Thema, die durch explizite oder implizite text- oder kontextsemantisch erschließbare Verweisungen aufeinander Bezug nehmen bzw. einen intertextuellen Zusammenhang bilden, an dem sich verschiedene Personen und Institutionen mit verschiedenen Wissensbeständen beteiligen, und der sich über einen längeren Zeitraum erstreckt.

Für die Verwendung im unterrichtlichen Kontext ist dieser Begriff noch zu weit gefasst, und es bedarf einiger Akzentuierungen. Diese können mit Jung und Karg vorgenommen werden. Jung gliedert drei Ebenen des Gesamtdiskurses „ $\mathrm{D}^{\prime \prime}$ auf: Auf der ersten Ebene „A“ spezifiziert er den Diskurs nach den Kommunikationsbereichen - "A1, A2 ... An" - beispielsweise politische Gruppen, Fachwissenschaften, die zweite Ebene „B“ stellen die inhaltlich abgrenzbaren Teildiskurse dar - „B1, B2 ... Bn“. Sie bilden die vertikale Gliederung. Die dritte Ebene "C $\mathrm{C}^{\prime}$ konstituieren solche Parameter wie Redekonstellationen bzw. Textsorten - „C1, C2, ... Cn“. Mit diesen Komponenten erstellt Jung ein Würfelmodell Jung (2000: 24). Ähnlich geht Karg vor und differenziert Diskurs auf dreifache Weise - als eine offene Menge von inhaltlich zusammenhängenden Texten, als die Art und Weise, wie eine Vielzahl von Themen zu verschiedenen Zeiten von bestimmten Diskursträgern verhandelt wird und an Textsorten und Textmustern orientiertes Diskursfeld (Karg 2007).

Anhand eines Beispiels kann dieser Zugang veranschaulicht werden. Gegenwärtig beschäftigen sich viele Wissenschaftler und Politiker mit der Verwendung in der Kinder- und Jugendliteratur aus heutiger Sicht diskri- 
minierender Ausdrücke. In diesem Fall ist das Thema Diskurs konstituierend. Alle Texte unabhängig von der Textart und Erscheinungsquelle, in denen sich Verfasser mit diesem Thema beschäftigen, konstituieren den Diskurs. Die Autoren des Geschriebenen beteiligen sich mit ihren Schriftstücken daran, führen es weiter oder verändern es sogar. Die Grenzen zu anderen Themenfeldern sind fließend, beispielsweise lassen sich Überschneidungen zum Diskurs um das Thema "Fremdenfeindlichkeit" feststellen. Es ist daher unmöglich, den Gesamtdiskurs zu fassen. Ein Beispiel des Diskurses als eine Art und Weise, wie eine Vielzahl von Themen zu verschiedenen Zeiten von bestimmten Diskursträgern verhandelt wird, bildet der Diskurs von Pädagogen. In diesem Fall führt eine bestimmte Gruppe von Spezialisten den Diskurs. Um die Diskursivität von Äußerungen als Möglichkeit, Themen jeder Art $\mathrm{zu}$ verhandeln $\mathrm{zu}$ veranschaulichen, ist beispielsweise an den Lyrikdiskurs zu denken, der dem rational-argumentativen Diskurs gegenüberzustellen ist, und sich von ihm durch mehrere Merkmale unterscheidet. Als Differenzierungsmerkmale sind Themen, Textmuster und Themenentwicklungen zu nennen.

Konkret für die unterrichtliche Vorgehensweise bedeutet das, einen Diskursausschnitt abzugrenzen. Dabei wird er je nach der anvisierten Zielsetzung und Lerngruppe variieren, d.h., wenn als Ziel des Unterrichts das Verfassen von Texten zu einem bestimmten Thema, beispielsweise „Fremdenfeindlichkeit und Rassismus" gesetzt wird, so ist der Diskurs mit diesem Schwerpunkt in den unterrichtlichen Rahmen hineinzuholen, d.h., Diskurs um dieses Thema, der sich in unterschiedlichen Texten, manifestiert beispielsweise in Interviews, Kommentaren, wissenschaftlichen Artikeln, (kinder)literarischen Texten, Beiträgen aus Foren und sogar aus anderen Medien beispielsweise Kurzfilmen usw. (Hier würde sich besonders gut der Kurzfilm „Der Schwarzfahrer" von Pepe Danquart aus dem Jahr 1992 eignen). Weiterhin gilt es, den Diskursausschnitt zu erkunden, ihn im unterrichtlichen Rahmen aufzubauen und zu rezipieren. Die Rezeption und die Produktion stehen in einer Wechselwirkung zueinander. Das Verfassen wird erst nach der Rezeption von Prätexten, d.h., dem eigenen Text vorausgegangenen Texten möglich. Dies implizit, dass im Unterricht bestimmte Normen, Konventionen und Vorstellungen, die eine bestimmte Sprechergemeinschaft im Laufe der Zeit entwickelt hat bzw. die sich etabliert haben, erkundet werden sollen. Der Erkundungsprozess ist sowohl für den muttersprachlichen als auch für den fremdsprachlichen Unterricht insofern von Bedeutung, weil die Lernenden bei der Textproduktion versuchen in der anvisierten Zielkultur diskursfähig zu werden. Die beschriebene Vorgehensweise kann in einem Modell folgendermaßen dargestellt werden. (S. Abbildung 1) 


\section{SCHREIBUNTERRICHT DISKURSFÄHIGKEIT UND INTERNET. EIN UNTERRICHTSBEISPIEL}

Die Entwicklung der Schreibfertigkeit nach dem vorgeschlagenen Prinzip wird nun anhand der sprachlichen Handlung des Argumentierens aufgezeigt. Es ist bereits an einer anderen Stelle angesprochen worden, dass hier kein konkretes Konzept aufgelegt, sondern eher eine modellhafte Vorgehensweise veranschaulicht wird.

Die Fähigkeit zu argumentieren wird sowohl im muttersprachlichen als auch im fremdsprachlichen Unterricht angestrebt (exemplarisch Kerncurriculum für das Gymnasium Schuljahrgänge 5-10, Niedersachsen und Angaben im GERR).

In den Unterrichtswerken findet man zwecks ihrer Entwicklung häufig folgende Regeln: These - Argumente dafür - Beispiele, Argumente dagegen Beispiele. Die Verhandlung eines Sachverhalts wird mit der eigenen Meinung bzw. Schilderung der eigenen Situation abgeschlossen. Im wirklichen Leben argumentiert man anders: Man versucht den Kommunikationspartner von bestimmten Sachverhalten zu überzeugen. Folglich wird eine These aufgestellt und mittels Argumente begründet. Es ist zwar richtig, dass auch die Gegenargumente erwähnt werden können, doch unterstützen sie nicht eine Antithese, die gleichwertig in den Text eingebaut ist. Diese und somit das Widerlegen der aufgestellten Behauptung erfolgen in einem anderen Schriftstück und in der Regel von einer anderen Person. Verbindung von These, Antithese und eigener Meinung in einem Text ist nur dann in der Wirklichkeit anzutreffen, wenn in den ersten beiden Abschnitten Fremdpositionen wiedergegeben werden. Die Thesen dieser Fremdpositionen werden als Hypothesen in den ersten beiden Teilen dargestellt, mit denen sich der Schreibende im letzten Teil auseinandersetzt, die er zu bewiesen oder zu widerlegen versucht. Auch das Abschließen einer Argumentation mit der Äußerung eigener Meinung ist eher wirklichkeitsfremd. Dies kommt häufiger in Kommentaren und Lesebriefen vor. Nimmt man als Ziel des Unterrichts vor, dass die Lernenden in der deutschen Sprache und entsprechend der in der Zielkultur verbreiteten Vorstellungen argumentieren lernen und an den kontroversen Verhandlungen der Wirklichkeit teilnehmen können, so gilt es, in der Wirklichkeit vorkommende Argumentationen in den Unterricht hereinzuholen. Das kann unter anderem mittels Internet geschehen. In diesem Medium findet man ein unbegrenztes Reservoir an Texten, in denen Themen kontrovers verhandelt werden, beispielsweise Artikel in OnlineVersionen von Zeitungen und Zeitschriften, Kunstkritiken und Leserbriefe, Rezensionen, Kommentare, normative Schriftstücke wie Gerichtsentscheidungen und wissenschaftliche Abhandlungen aber auch appellative Schriftstücke wie Werbetexte. Zudem ist an weitere Vorteile zu denken, nämlich an den Gegenwartsbezug des Veröffentlichten sowie seine Authentizität. 


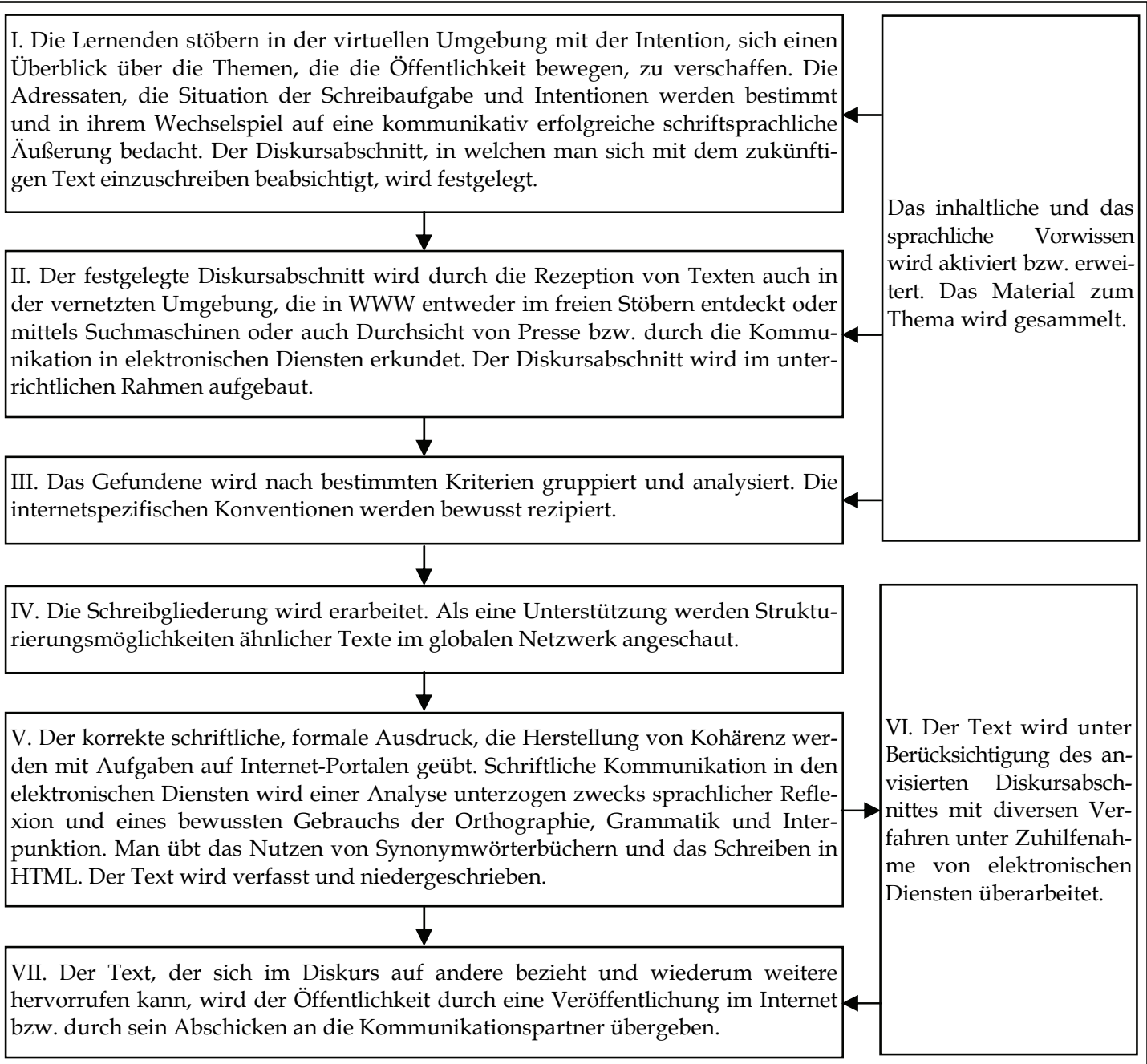

Abb. 1. Modell des Schreibens mit dem Internet Kuzminykh (2009: 199)

Also werden im ersten Schritt Themen, die zum Zeitpunkt des Schreibunterrichts die Öffentlichkeit bewegen, erkundet. Entweder mit den Suchwerkzeugen des globalen Netzes oder unmittelbar auf den Web-Seiten von digitalen Zeitungen und Zeitschriften sucht man nach Artikeln, in denen die Verfasser durch Angabe von Gründen von ihrer Sichtweise bzw. von ihrer Wertung eines Sachverhalts den Lesenden zu überzeugen versuchen, und ihn gegebenenfalls zu einem entsprechenden Verhalten veranlassen. So bekommen die Erkundenden eine Vorstellung von dem, was die jeweilige, 
anvisierte Sprechergemeinschaft bewegt und von ihren Normen und Werten. Dies ermöglicht es, den Rahmen der unterrichtlichen Wirklichkeit zu durchbrechen, und den Bezug zu Realität herzustellen. Unterrichtspraktisch lässt sich diese Arbeit in Gruppen durchführen. Die Themen werden im Unterricht besprochen, und die Lernenden entscheiden sich, mit welchem Thema sie sich argumentativ auseinandersetzen wollen. In einem weiteren Schritt werden die auf das vereinbarte Thema bezogenen Argumentationen herausgesucht. Auch dies lässt sich in Gruppen- bzw. in Partnerarbeit verwirklichen. Es kann hier an dem Thema „Rassismus und Fremdenfeindlichkeit" veranschaulicht werden. Eine Zusammenstellung von Internaderessen $\mathrm{zu}$ diesem Thema ist im Literaturverzeichnis zu finden. Das Gefundene unterzieht man mittels einfacher argumentationstheoretischer Begriffe, nämlich These, Argument und Schlussregel einer Analyse (Toulmin 1958). Ein Beispiel:

These: Die diskriminierenden Ausdrücke „Neger“, „Negerlein“, „Mohren“ „Zigeuner" und Ähnliche sollen aus Kinderbüchern entfernt werden.

Argument: Diskriminierende Begriffe fließen ungefiltert in Sprachgebrauch von Kindern im Vorlesealter, die noch nicht differenzieren können, ein.

Schlussregel: Wenn die diskriminierenden Begriffe ungefiltert in Sprachgebrauch von Kindern im Vorlesealter, die noch nicht differenzieren können, einfließen, dann sollen die diskriminierenden Ausdrücke "Neger", "Negerlein“, "Mohren“ "Zigeuner" und Ähnliche aus Kinderbüchern entfernt werden.

Der Erkundungsprozess und Analyse logischer Beziehungen in kontroversen schriftsprachlichen Verhandlungen ermöglichen, sprachliche Reflexion sinnvoll in den Lernprozess einzubeziehen. Dabei handelt es sich um die Verwendung von Konjunktionen mit kausaler und konditionaler Bedeutung. Ihr Gebrauch begründet sich, und die Lernenden müssen diese nicht ohne Verwendungszusammenhang üben. Dies betrifft sowohl den fremdsprachlichen als auch muttersprachlichen Deutschunterricht.

Weiterhin sollen die Grundlage für die Überzeugungskraft von Argumenten und ihre Relativität untersucht werden. Die Textverfasser können dafür sensibilisiert werden, dass es keine allgemeingültigen Kriterien für das Erreichen der Plausibilität gibt, sondern sie vom Vorwissen des Lesenden, seinen Wertvorstellungen und Überzeugungen abhängt. Dies lässt sich an einem argumentativen Schriftstück aus der Realität üben: Es wird ein Text rezipiert und Argumente, die von den Schreibenden als besonders plausibel eingeschätzt werden, ausgegliedert. Anschließend präsentiert man das Ausgeschriebene im Plenum. Die Lernenden werden unterschiedliche Begründungen, die sie als akzeptabel erachten, entsprechend Wissensstand aus dem Text entnommen haben. Sich diese Tatsache vor Augen zu führen, kann 
die Abhängigkeit der Beweismittel von den Wissensbeständen und Überzeugungen eines Rezipienten verdeutlichen. Anschließend können die Lernenden entscheiden, welche Position sie in den eigenen schriftlichen Äußerungen vertreten wollen, und an wen sie sich mit ihren eigenen argumentativen Schriftstücken wenden. Das Letztere ist insofern relevant, weil die Textautoren vom Vorhandensein bestimmter Hintergrundkenntnisse bei den zukünftigen Rezipienten ausgehen und entsprechend diesen Vorstellungen ihre Argumente auswählen können. Die kulturellen und sozialen Normvorstellungen sowie die Wissensbestände von dem, was eine bestimmte Gruppe von Rezipienten für einleuchtend und überzeugend hält, können im Unterricht konkretisiert werden. Somit steigt die Chance auf Erfolg des argumentativen Schriftstücks. Nun können die Gliederungen erstellt und eigene Texte verfasst werden.

Die Überarbeitung kann so erfolgen, dass das Geschriebene an Klassenpartner per elektronische Post direkt bzw. anonym über den Account des Lehrers versendet wird. Die Empfänger lesen das Eingegangene und verfassen einen knappen Kommentar darüber, ob die Argumente so ausformuliert sind, dass sie nachvollzogen werden können, und ob sich zwischen der These, ihren Begründungen und der Schlussregel logische Beziehungen herstellen lassen. Schließlich verbessern die Lernenden ihre Texte und können sie der Öffentlichkeit im Internet übergeben. Das Geschriebene kann somit in einem bestimmten Diskusfeld eine Bedeutung haben.

Wird die Fähigkeit zu argumentieren so vermittelt, kommt der Lehrperson eine besondere Rolle zu. Ihre Aufgabe besteht in der Gewährleistung individueller Hilfestellungen in Bezug auf Recherche von Texten und bei der Aufstellung von These sowie im Finden von angemessenen Argumenten, in der Anwendung von Suchtechniken auch im Medium Internet und in der Beseitigung von Verständnisschwierigkeiten, in der Unterstützung bei der Anwendung von Schreibstrategien und Regeln der Argumentation, d.h., in der Formulierung der Schlussregeln sowie in individuellen Formulierungshilfen.

\section{ZUSAMMENFASSUNG}

Eine solche Vorgehensweise kann bei der Entwicklung von unterschiedlichsten schriftsprachlichen Handlungen - Beschreiben, Erzählen, Verfassen von Zusammenfassungen, Rezensionen und Briefen - eingesetzt werden. Es ermöglicht, Voraussetzungen für die Entstehung von Texten zu schaffen, die in einem (Diskurs)zusammenhang zu den vorausgegangenen Schriftstücken stehen und auch Nachtexte hervorrufen könnten. Sukzessiv werden die Ler- 
nenden an das Verfassen von solchen Texten herangeführt, mit denen sie sich an der schriftsprachlichen Kommunikation einer muttersprachlichen und einer fremden Gesellschaft beteiligen könnten, da sie kontinuierlich daran und damit arbeiten. Die Vermittlung von sprachlichen Fähigkeiten und Fertigkeiten, nämlich Sprach-, Text-, Situations-, Handlungs-, und Weltwissens einschließlich des Wissens von pragmatischen, stilistischen und sozialen Konventionen, die in einer Sprechergemeinschaft entwickelt sind, begründet sich daraus. Sie erfolgt vor dem Hintergrund des Diskurses. Vielmehr: Die auf diese Art und Weise erworbene Schreibfertigkeit lässt sich sehr wohl außerhalb der unterrichtlichen Umgebung zur Bewältigung unterschiedlicher Wirklichkeitssituationen anwenden. Ein weiterer Vorteil besteht in der Förderung der Medienkompetenz.

\section{LITERATURVERZEICHNIS}

Adamzik, K., 2004a. Sprache: Wege zum Verstehen. Tübingen: Francke.

Adamzik, K., 2004b. Textlinguistik. Eine einführende Darstellung. Tübingen: Niemeyer.

Beck, O., 1970. Aufsatzerziehung und Aufsatzunterricht. Ein Lehrerhandbuch. Band 1, 1.-4. Schuljahr. Bonn-Bad Godesberg: Dürr.

Beck, O., 1971. Aufsatzerziehung und Aufsatzunterricht. Ein Lehrerhandbuch. Band 2, 5.-9. Schuljahr. Bonn-Bad Godesberg: Dürr.

Bluhm, C., Deissler, D., Scharloth, J., Stukenbrock, A., 2000. Linguistische Diskursanalyse: Überblick, Probleme, Perspektiven. In: Sprache und Literatur in Wissenschaft und Unterricht, 31, 3-19.

Brenner, G., 1998. Kreatives Schreiben. Ein Leitfaden für die Praxis. Frankfurt am Main: Cornelsen.

Busse, D., Teubert, W., 1994. Ist Diskurs ein sprachwissenschaftliches Objekt? Zur Methodenfrage der Historischen Semantik. In: Busse, D., Hermanns, F., Teubert, W. (Hrsg.). Begriffsgeschichte und Diskursgeschichte: Methodenfragen und Forschungsergebnisse der historischen Semantik. Opladen: Westdeutscher Verlag, 10-28.

Fritzsche, J., 1980. Aufsatzdidaktik. Kritische und systematische Untersuchungen zu den Funktionen schriftlicher Texte von Schülern. Stuttgart: Kohlhammer.

Fröchling, J., 1990. Kreatives Schreiben und Individuum. In: Ermert, K. (Hrsg.). Was bewegt die Schreibbewegung. Loccumer Protokolle. Loccum: Evangelische Akademie Loccum (Selbstverlag).

Haueis, E., Hoppe, O., 1972. Aufsatz und Kommunikation. Zwei Untersuchungen. Düsseldorf: Schwann.

Hayes, J.R., Flower, L., 1980. Identifying the organization of writing processes. In: Gregg, L., Steinberg, E.R. (Hrsg.). Cognitive Prozesses in Writing. Hillsdale, NJ: Erlbaum, 3-10.

Helmers, H., 1972. Didaktik der deutschen Sprache. Einführung in die Theorie der muttersprachlichen und literarischen Bildung. Stuttgart: Klett.

Huneke, H.-W., Steinig W., 2002. Deutsch als Fremdsprache: eine Einführung. Berlin: Schmidt.

Jung, M., 2000. Diskurshistorische Analyse als linguistischer Ansatz. In: Sprache und Literatur in Wissenschaft und Unterricht, 31, 20-39.

Karg, I., 2007. Diskursfähigkeit als Paradigma schulischen Schreibens. Ein Weg aus dem Dilemma zwischen Aufsatz und Schreiben. Frankfurt am Main: Lang. 
Krumm, H.-J., 1994. Mehrsprachigkeit und interkulturelles Lernen. Orientierungen am Fach Deutsch als Fremdsprache. In: Jahrbuch Deutsch als Fremdsprache, 20, 13-36.

Kruse, O., Perrin, D., 2003. Intuition und professionelles Schreiben. In: Perrin, D., Böttcher, I., Kruse, O., Wrobel, A., (Hrsg.). Schreiben. Von intuitiven zu professionellen Schreibstrategien. Wiesbaden: Westdeutscher Verlag, 5-25.

Kuzminykh, K., 2009. Das Internet im Deutschunterricht. Ein Konzept der muttersprachlichen und der fremdsprachlichen Lese- und Schreibdidaktik. Frankfurt am Main: Lang.

Ludwig, O., 1988. Der Schulaufsatz. Seine Geschichte in Deutschland. Berlin: de Gruyter.

Ludwig, O., 2003. Entwicklung der schulischen Schreibdidaktik und ihr Bezug zum akademischen Schreiben. In: Ehlich, K., Steets, A. (Hrsg.). Wissenschaftlich schreiben lehren und lernen. Berlin: de Gruyter, 235-250.

Marthaler, T., 1962. Es gibt sechs Aufsatzarten. In: Der Deutschunterricht, 14/4, 53-63.

Neufeld, D., 2013. Ich die Negerin. In: Spiegel, 4, 57.

Neuner, G., Hunfeld, H., 2004. Methoden des fremdsprachlichen Deutschunterrichts: Eine Einführung. Berlin: Langenscheidt.

Portmann, P.R., 1991. Schreiben und Lernen. Grundlagen der fremdsprachlichen Schreibdidaktik. Tübingen: Niemeyer.

Roche, J., 2001. Interkulturelle Sprachdidaktik. Eine Einführung. Tübingen: Narr.

Scheidt, J., 1993. Kreatives Schreiben. Wege zu sich selbst und zu anderen. Frankfurt am Main: Fischer.

Spinner, K., 1993. Kreatives Schreiben. In: Praxis Deutsch, 119, 17-23.

Stenschke, O., 2005. Rechtschreiben, Recht sprechen, recht haben - der Diskurs über die Rechtschreibreform: eine linguistische Analyse des Streits in der Presse. Tubingen: Niemeyer.

Toulmin, S., 1958. The Uses of Argument. Cambridge: Cambridge University Press.

Vater, H., 2002. Einführung in die Sprachwissenschaft. München: Fink.

\section{Nachschlagewerke}

Gemeinsamer europäischer Referenzrahmen für Sprachen (GERR): lernen, lehren, beurteilen. 2001. Goethe-Institut Inter Nationes. Berlin: Langenscheidt.

Kerncurriculum für das Gymnasium Schuljahrgänge 5-10 Deutsch Niedersachsen http://db2.nibis. de/1db/cuvo/datei/kc_gym_deutsch_nib.pdf (abgerufen am: 29.01.2013).

\section{Unterrichtswerke}

Biermann, et. al. 1998. Deutschbuch 10. Berlin: Cornelsen.

Biermann, et. al. 2000. Deutschbuch 10. Arbeitsheft. Berlin: Cornelsen.

Braun, et. al. 2000. Unterwegs zur Vorbereitung auf die Zentrale Mittelstufenprüfung. Trainingsbuch. Berlin: Langenscheidt.

Dallapiazza, et. al. 2003. Tangram Aktuell Zertifikatsband. Ismaning: Hueber.

Dallapiazza, et. al. 2008. Ziel B2. Bd. 1. Paket Kursbuch und Arbeitsbuch mit Lerner-Audio-CD/CDROM. Ismaning: Hueber.

Köhl-Kuhn, et. al. 2008. Mittelpunkt B2. Lehrbuch Deutsch als Fremdsprache für Fortgeschrittene. Stuttgart: Klett.

Köhl-Kuhn, et. al. 2008. Mittelpunkt C1. Lehrbuch Deutsch als Fremdsprache für Fortgeschrittene. Stuttgart: Klett.

Müller, et. al. 2005. Kombi-Buch Deutsch 6 Schulbuch für den Unterricht. Bamberg: Buchner.

Müller, et. al. 2006. Kombi-Buch Deutsch 8 Schulbuch für den Unterricht. Bamberg: Buchner. 
Niebisch, et. al. 2009. Schritte plus 1. Kursbuch und Arbeitsbuch mit Audio-CD zum Arbeitsbuch. Ismaning: Hueber.

Rohrmann et. al. 2004. Berliner Platz 3. Berlin: Langenscheidt.

Rohrmann et. al. 2005. Berliner Platz 1. Berlin: Langenscheidt.

\section{Internetquellen}

Amrhein, M. Schuldig sind wir alle“. http://www.cicero.de/salon/neger-preu\%C3\%9Flerentschuldigung-ich-habe-das-n-wort-gesagt/53195 (abgerufen am: 18.02.2013).

Forum zum Thema Rassismus in Kinderbücher. http://forum.gofemi-nin.de/forum/couple1 /_f86697_couple1-OT-Rassismus-in-Kinderbuchern.html (abgerufen am: 18.02.2013).

Halser, A. Igel bratende Zigeuner. http://www.taz.de/!66388/ (abgerufen am: 18.02.2013).

Hein, J. Werte und Worte von. http://www.taz.de/!109029/(abgerufen am: 18.02.2013).

John, B. Verbale Salzsäure. http://www.tagesspiegel.de/meinung/rassismus-in-kinderbuechernverbale-salzsaeure-/7693104.html (abgerufen am: 18.02.2013).

Kinderbücher: Mehrheit will keine Änderungen. http://www.bz-berlin.de/kultur/literatur/ kin derbuecher-mehrheit-will-keine-aenderungen-article1636779.html (abgerufen am: 18.02.2013).

Kinderbücher ohne Rassismus. http://www.dw.de/kinderb\%C3\%BCcher-ohne-rassismus/a16532208 (abgerufen am: 18.02.2013).

Martenstein, H. „Achtung, Zensur“. http://www.tagesspiegel.de/meinung/rassismus-in-kinder buechern-achtung-zensur/7658452.html (abgerufen am: 18.02.2013).

Negerkönig sorgt für Ärger. http://www.n-tv.de/panorama/Negerkoenig-sorgt-fuer-Aergerarticle2696131.html

Rassismus raus aus den Kinderbüchern. http://maedchenmannschaft.net/rassismus-raus-auskinderbuechern/ (abgerufen am: 18.02.2013).

Schwarze Kinder als Helden und Heldinnen im Kinderbuch Bücherausstellung, Vortrag im Kinderladen Maimouna unter. http://www.maimounakila.de/pdf/Bericht-von-den-Lesetagebei-Maimouna.pdf (abgerufen am: 18.02.2013).

Sprachliche Modernisierung von Klassikern - offizielle Stellungnahme des Thienemann Verlags. http://cms.thienemann.de/index.php? $\neg$ option=com_content\&view=article\&id=637:sprach liche-modernisierung-von-klassikern\&cat $\neg$ id=15:news-artikel\&Itemid=29 (abgerufen am: 18.02.2013).

Voigts, H. Worte können wehtun. http://www.fr-online.de/frankfurt/rassistische-begriffe-inkinderbuechern-worte-koennen-wehtun,1472798,21587554.html (abgerufen am: 18.02.2013). 
